\title{
On the Boundary of the Union of Planar Convex Sets*
}

\author{
J. Pach ${ }^{1,2,3}$ and M. Sharir ${ }^{2,4}$ \\ ${ }^{1}$ Department of Computer Science, City College, CUNY, \\ New York, NY 10031, USA \\ ${ }^{2}$ Courant Institute of Mathematical Sciences, New York University, \\ New York, NY 10012, USA \\ pach@cims.nyu.edu \\ ${ }^{3}$ Hungarian Academy of Sciences, Budapest, Hungary \\ ${ }^{4}$ School of Mathematical Sciences, Tel Aviv University, \\ Tel Aviv 69978, Israel \\ sharir@math.tau.ac.il
}

\begin{abstract}
We give two alternative proofs leading to different generalizations of the following theorem of [1]. Given $n$ convex sets in the plane, such that the boundaries of each pair of sets cross at most twice, then the boundary of their union consists of at most $6 n-12$ arcs. (An arc is a connected piece of the boundary of one of the sets.) In the generalizations we allow pairs of boundaries to cross more than twice.
\end{abstract}

\section{Introduction}

Let $\mathcal{C}$ be a collection of $n \geq 3$ nondegenerate convex sets (bodies) in the plane, any two of which have at most a finite number of boundary points in common. Assume for simplicity that the sets are in general position, i.e., no two boundary curves are tangent to each other, and no three pass through the same point. If two members of $\mathcal{C}$ have exactly two boundary points in common, then these points are called regular vertices of the

\footnotetext{
* Work on this paper by both authors has been supported by NSF Grant CCR-94-24398. Work by János Pach was also supported by Grant OTKA-T-020914 and by a CUNY Research Award. Work by Micha Sharir was also supported by NSF Grant CCR-93-11127, by the Hermann Minkowski-MINERVA Center for Geometry, and by grants from the U.S.-Israeli Binational Science Foundation, and the G.I.F., the German-Israeli Foundation for Scientific Research and Development.
} 


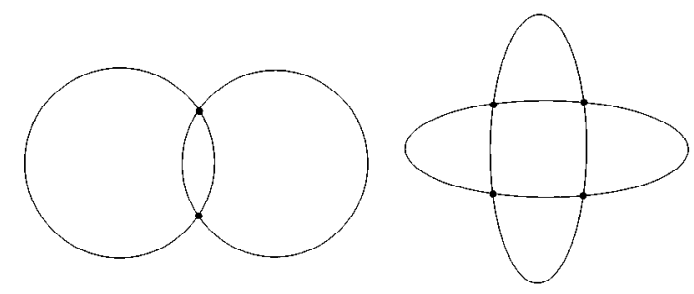

Fig. 1. Regular vertices are shown on the left, and irregular vertices on the right.

arrangement $\mathcal{A}(\mathcal{C})$. All other intersection points of the boundary curves are said to be irregular. See Fig. 1.

Let $U=\bigcup \mathcal{C}$ denote the union of all members of $\mathcal{C}$. Let $R$ and $I$ denote the set of regular and irregular vertices of $\mathcal{A}(\mathcal{C})$, respectively, lying on $\partial U$, the boundary of $U$. Further, put $V=R \cup I$. If the sets in $\mathcal{C}$ are bounded, then $|V|$ is equal to the number of arcs that compose $\partial U$.

It was shown in [1] that if any two members of $\mathcal{C}$ have at most two boundary points in common (i.e., if there are no irregular vertices), then $|R|=|V| \leq 6 n-12$, and this bound is tight in the worst case. In Section 2 of this note, we generalize this result as follows:

Theorem 1. With the above notation, for any collection of $n \geq 3$ nondegenerate convex sets in general position in the plane satisfying the above assumptions, we have

$$
|R| \leq 2|I|+6 n-12 \text {. }
$$

Actually, in [1] the members of $\mathcal{C}$ were not required to be convex, and it is very likely that Theorem 1 also generalizes to that case.

Whitesides and Zhao [4] introduced the following definition. A collection of closed Jordan curves is called $k$-admissible if no two curves touch each other, any two curves intersect in at most $k$ points, and the interior of no curve disconnects the interior of another. Clearly, we can restrict our attention to the case when $k$ is even. In Section 5 we give a new proof of the following result of [4], which provides yet another generalization of the above mentioned theorem of [1].

Theorem 2. The number of vertices on the boundary of the union of the interiors of $n \geq 3$ Jordan curves that form a $k$-admissible family, is at most $k(3 n-6)$; this bound is tight in the worst case.

The methods used here are quite different from those used in [1] and [4].

\section{Proof of Theorem 1}

Preliminaries. We can assume without loss of generality that every member of $\mathcal{C}$ is bounded and that its boundary is smooth. It is sufficient to establish the theorem in 
the case when $U=\bigcup \mathcal{C}$ is connected; otherwise, arguing for each component of $U$ separately, we obtain the stronger inequality $|R| \leq 2|I|+6 n-12 k_{\geq 3}-10 k_{2}-6 k_{1}$, where $k_{1}$ (resp. $k_{2}, k_{\geq 3}$ ) is the number of connected components of $U$ formed by one (resp. two, at least three) sets of $\mathcal{C}$.

A connected component $H$ of the complement of $U$ is called a hole. Let $V(H)$ denote the set of vertices along the boundary of a hole $H$. These vertices divide the boundary of $H$ into $|V(H)|$ arcs, which form a set denoted by $\Gamma(H)$. The set of all arcs composing $\partial U$ is denoted by $\Gamma_{\text {ext }}=\bigcup_{H} \Gamma(H)$. Note that every bounded hole has at least three vertices. The unique unbounded hole may have fewer vertices (zero or two), but then $|V| \leq 2$. We may therefore assume that every hole has at least three vertices, so the number $h$ of holes is at most $|V| / 3$.

Orient the boundary of every $c \in \mathcal{C}$ in the counterclockwise direction. Accordingly, every (unit) tangent vector to $c$ will be oriented so that $c$ lies on its left-hand side.

Consider now two sets $c, c^{\prime} \in \mathcal{C}$ whose boundaries intersect in exactly two points $v$ and $v^{\prime}$. (These are regular vertices of the arrangement.) Then $c \cap c^{\prime}$ is a lens-like region, whose boundary is a counterclockwise oriented closed curve $\xi_{c c^{\prime}}$, with the two "breakpoints" (nonsmooth points) $v$ and $v^{\prime}$. Denote the turning angles of (the tangents to) $\xi_{c c^{\prime}}$ at $v$ and $v^{\prime}$ by $a(v)$ and $a\left(v^{\prime}\right)$, respectively. (Note that $a(v), a\left(v^{\prime}\right)$ are always positive. See Fig. 2.) A similar definition applies when the boundaries of $c$ and $c^{\prime}$ meet irregularly at $v$ : we then define $a(v)$ to be the turning angle of the boundary of $c \cap c^{\prime}$ at $v$.

Total Turning Angles of Piecewise Smooth Curves. Let $\xi$ be an oriented continuous curve in the plane. If at some point $w$ of $\xi$, there is no unique tangent line, then $w$ is called a breakpoint. We say that $\xi$ is piecewise smooth, if it has finitely many breakpoints, and every piece of $\xi$ between two consecutive breakpoints is differentiable (including at its endpoints).

Define the total turning angle $\theta(\xi)$ of a piecewise smooth, oriented curve $\xi$ as follows. If necessary, subdivide $\xi$ into smaller differentiable oriented arcs $\xi_{1}, \ldots, \xi_{m}$, such that each $\xi_{i}$ is smooth and any two tangents to the same arc $\xi_{i}$, oriented according to the orientation of the curve, differ in their orientations by less than $\pi$. Let $-\pi<\theta\left(\xi_{i}\right)<+\pi$

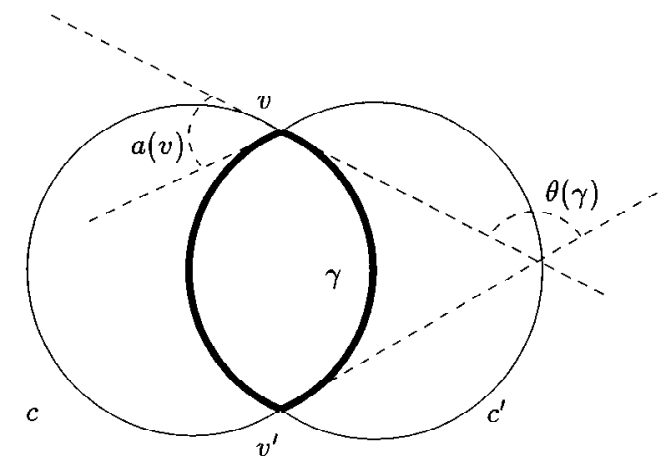

Fig. 2. Two sets $c, c^{\prime}$ intersecting regularly, and the curve $\xi_{c c^{\prime}}$, shown in bold. Also shown are the turning angle $\theta(\gamma)$ along the curve $\gamma$ and the turning angle $a(v)$ at the vertex $v$. 
be the smaller angle from the tangent vector at the starting point of $\xi_{i}$ to the tangent vector at the endpoint of $\xi_{i}$, taken with positive sign if the change is counterclockwise and with negative sign otherwise (see, e.g., Fig. 2). At each point $w_{i}$ separating two pieces, $\xi_{i}$ and $\xi_{i+1}$, let $\theta\left(w_{i}\right)$ be the smaller angle from the tangent to $\xi_{i}$ at $w_{i}$ to the tangent to $\xi_{i+1}$ at $w_{i}$, with positive sign if and only if it is counterclockwise. If $w_{i}$ is not a breakpoint, then, by construction, $\theta\left(w_{i}\right)=0$. Finally, let the total turning angle $\theta(\xi)$ be defined as the sum of $\theta\left(\xi_{i}\right)$ over all pieces $\xi_{i}$ plus the sum of $\theta\left(w_{i}\right)$ over all vertices $w_{i}$. Evidently, this definition of the turning angle is independent of the particular subdivision of $\xi . \theta\left(\xi_{i}\right)$ and $\theta\left(w_{i}\right)$ are called, respectively, the turning angle of $\xi$ along the arc $\xi_{i}$ and at the point $w_{i}$.

The following lemma summarizes the elementary properties of the total turning angle. We omit the trivial proof.

Lemma 3. Let $\xi$ be a piecewise smooth, oriented curve in the plane with total turning angle $\theta(\xi)$.

(i) If $\xi$ is a closed curve, then $\theta(\xi)$ is an integer multiple of $2 \pi$.

(ii) If $\xi$ is a counterclockwise (resp. clockwise) oriented closed curve which does not intersect itself, then $\theta(\xi)=2 \pi$ (resp. $-2 \pi)$.

(iii) If $\xi$ intersects itself at a point $w$, then it can be decomposed into two piecewise smooth, oriented curves, $\xi^{\prime}$ and $\xi^{\prime \prime}$, having the common breakpoint $w$. (If $\xi$ is a closed curve, then so are $\xi^{\prime}$ and $\xi^{\prime \prime}$; if $\xi$ is open, then one of the two parts is open and the other is closed.) In both cases we have

$$
\theta(\xi)=\theta\left(\xi^{\prime}\right)+\theta\left(\xi^{\prime \prime}\right)
$$

We refer to the last equality as the additivity property of the total turning angle.

Turning Angles Along Holes of the Union. Notice that the orientation of the boundary of any bounded hole $H$ of $U$ is clockwise, and the orientation of the boundary of the unique unbounded hole is counterclockwise. At any regular vertex $v$ on the boundary of any hole, the turning angle of the boundary is $-a(v)$. Thus, Lemma 3(ii) implies that, for any fixed bounded hole $H$,

$$
\sum_{v \in V(H)}(-a(v))+\sum_{\gamma \in \Gamma(H)} \theta(\gamma)=-2 \pi,
$$

and, for the unique unbounded hole, the left-hand side is equal to $2 \pi$. Adding all these equations, multiplying by -1 and ignoring terms $a(v)$ for $v \in I$, we obtain

$$
\sum_{v \in R} a(v)-\sum_{\gamma \in \Gamma_{\mathrm{ext}}} \theta(\gamma) \leq 2 \pi(h-1)-2 \pi=2 \pi h-4 \pi \leq \frac{2 \pi(|R|+|I|)}{3}-4 \pi .
$$

Let $\Gamma_{\text {int }}$ denote the collection of maximal boundary arcs of the sets in $\mathcal{C}$, oriented as above, that are contained in the interior of $U$. In the next section we establish the following lemma.

\section{Lemma 4.}

$$
\sum_{v \in R}(\pi-a(v)) \leq \sum_{\gamma \in \Gamma_{\text {int }}} \theta(\gamma)
$$


It is easy to see that Lemma 4 implies Theorem 1. Indeed, the right-hand side of (2) is equal to $2 \pi n-\sum_{\gamma \in \Gamma_{\text {ext }}} \theta(\gamma)$. Summing up (1) and (2), we obtain

$$
\pi|R| \leq 2 \pi n+\frac{2 \pi(|R|+|I|)}{3}-4 \pi,
$$

which yields that $|R| \leq 2|I|+6 n-12$, as asserted.

\section{Proof of Lemma 4}

Let $\Gamma^{R}$ denote the subset of those arcs in $\Gamma_{\text {int }}$ that have at least one regular endpoint. The union of $\Gamma^{R}$ is decomposed into a collection of oriented cycles and paths; the vertices (breakpoints) of the cycles and the internal vertices of the paths belong to $R$, and the endpoints of the paths belong to $I$. In the next two subsections (A and B), we prove:

Claim. The total turning angle of each of these cycles and paths is at least $k \pi$, where $k$ is the number of all vertices of a cycle or the number of internal vertices of a path.

In subsection $\mathrm{C}$ we show how Lemma 4 follows from this fact.

A: The Case of a Cycle. Let $\zeta=v_{0} v_{1} \cdots v_{k}\left(v_{k}=v_{0}\right)$ be one of these oriented cycles, with vertices $v_{0}, v_{1}, \ldots, v_{k-1} \in R$. Let $\gamma_{i}$ denote the oriented arc along $\zeta$ connecting $v_{i-1}$ to $v_{i}$, and let $c_{i}$ be the set in $\mathcal{C}$ whose boundary contains $\gamma_{i}$, for $i=1, \ldots, k$.

We first consider the simple case $k=2$. In this case, $v_{0}$ and $v_{1}$ are the two (regular) intersections of $\partial c_{1}$ and $\partial c_{2}$, and $\zeta$ is the convex curve $\xi_{c_{1} c_{2}}$ defined above (see Fig. 2). Clearly, $\theta(\zeta)=2 \pi$, as claimed.

Next suppose that $k \geq 3$. We traverse $\zeta$ from $v_{0}$, and consider the tangents to $\zeta$, oriented in accordance with the orientation of $\zeta$ (so that the sets they are tangent to lie on their left). By construction, as we follow these tangents, they keep turning in the counterclockwise (positive) direction, and this also holds at each vertex of $\zeta$. See Fig. 3.

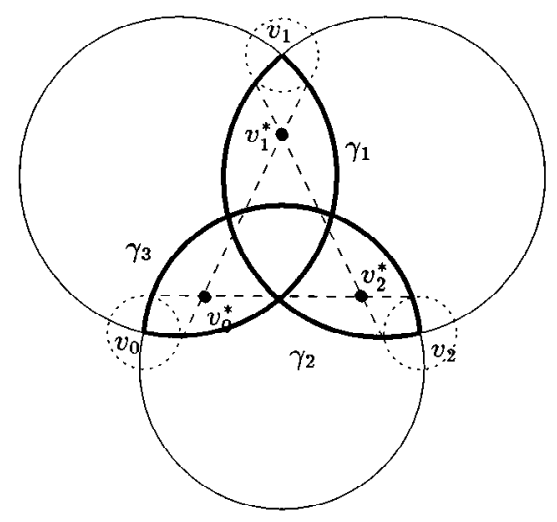

Fig. 3. Illustrating the proof of Lemma 4 for a cycle of $\Gamma^{R}$. 
For each $i=1, \ldots, k$, choose a very small $\varepsilon>0$, and draw a circle of radius $\varepsilon$ around each vertex $v_{i}$. Let $v_{i}^{-}$and $v_{i}^{+}$denote intersection points of this circle with $\gamma_{i}$ and $\gamma_{i+1}$, respectively (with $\gamma_{k+1}=\gamma_{1}$ ). Let $\zeta^{\prime}$ denote the closed curve obtained from $\zeta$ by replacing the portion of $\gamma_{i}$ between $v_{i-1}^{+}$and $v_{i}^{-}$by a straight-line segment, for every $i$. Clearly, the total turning angle of $\zeta^{\prime}$ is equal to the total turning angle of $\zeta$. See Fig. 3.

We claim that $\zeta^{\prime}$ can be decomposed into $k$ positively (i.e., counterclockwise) oriented loops at the vertices $v_{i}$ and an oriented closed polygon $\zeta^{*}=v_{0}^{*} v_{1}^{*} \cdots v_{k}^{*} ;\left(v_{k}^{*}=v_{0}^{*}\right)$. This follows from the fact that $v_{i-1}$ and $v_{i+1}$, the other endpoints of the arcs $\gamma_{i}$ and $\gamma_{i+1}$, lie on different sides of the line connecting $v_{i}$ and the other regular intersection point $v_{i}^{\prime}$ of the boundaries of $c_{i}$ and $c_{i+1}$. (Since $k \geq 3, v_{i}^{\prime}$ lies in the interior of the union, and $\gamma_{i-1}, \gamma_{i}$ cross each other at that point.) Consequently, if $\varepsilon$ is sufficiently small, then the segments $v_{i-1}^{+} v_{i}^{-}$and $v_{i}^{+} v_{i+1}^{-}$must cross each other in a small neighborhood of $v_{i}$, at a point denoted by $v_{i}^{*}$. The $i$ th loop of $\zeta^{\prime}$ is its portion that starts and ends at $v_{i}^{*}$. Again, see Fig. 3. Thus, by the additivity of the turning angle,

$$
\theta\left(\zeta^{\prime}\right)=k(2 \pi)+\theta\left(\zeta^{*}\right)
$$

By definition, at each vertex of $\zeta^{*}$, the absolute value of the turning angle of $\zeta^{*}$ is at most $\pi$ (and the turning angle along its edges is 0 ). Consequently, $\theta(\zeta)=\theta\left(\zeta^{\prime}\right) \geq k \pi$. (Actually, by Lemma 3(i), the total turning angle of $\zeta$ must be a multiple of $2 \pi$, so $\theta(\zeta) \geq 2\lceil k / 2\rceil \pi$ is also true. This is indeed the case shown in Fig. 3: the total turning angle of $\zeta$ is $4 \pi=2\left\lceil\frac{3}{2}\right\rceil \pi$.)

B: The Case of a Path. Consider now a path $\zeta=v_{0} v_{1} \cdots v_{k} v_{k+1}$ with irregular endpoints and regular internal vertices. Let $\gamma_{i}, c_{i}$, for $i=1, \ldots, k+1$, and $v_{i}^{-}, v_{i}^{+}$, for $i=1, \ldots, k$, denote the same entities as for cycles (the previous case). We also put $v_{0}^{+}=v_{0}$ and $v_{k+1}^{-}=v_{k+1}$. In exactly the same way as before, we construct a curve $\zeta^{\prime}$ from $\zeta$ by replacing with a straight-line segment the portion of $\gamma_{i}$ between $v_{i-1}^{+}$and $v_{i}^{-}$, for every $i=1, \ldots, k+1$. We have that $\theta(\zeta) \geq \theta\left(\zeta^{\prime}\right)$ (we turn more along $\gamma_{1}$ from $v_{0}$ to $v_{1}^{-}$than by going straight from $v_{0}$ to $v_{1}^{-}$and then turning at $v_{1}^{-}$until we are tangent to $\gamma_{1}$, and similarly at the other end of $\zeta$; see Fig. 4). Now, arguing as in the case of cycles, $\zeta^{\prime}$ is decomposed into $k$ positively oriented loops and a polygonal path $\zeta^{*}$. Again, the additivity of the turning angle implies that

$$
\theta(\zeta) \geq \theta\left(\zeta^{\prime}\right)=k(2 \pi)+\theta\left(\zeta^{*}\right)
$$

Since at each internal vertex of $\zeta^{*}$, the turning angle is between $-\pi$ and $+\pi$, we have that the total turning angle of $\zeta$ is at least $k \pi$.

C: Putting It Together. If $\zeta$ is a cycle, its total turning angle is, in the above notations,

$$
\sum_{i=1}^{k} a\left(v_{i}\right)+\sum_{i=1}^{k} \theta\left(\gamma_{i}\right) \geq k \pi,
$$

which implies that

$$
\sum_{i=1}^{k}\left(\pi-a\left(v_{i}\right)\right) \leq \sum_{i=1}^{k} \theta\left(\gamma_{i}\right) .
$$




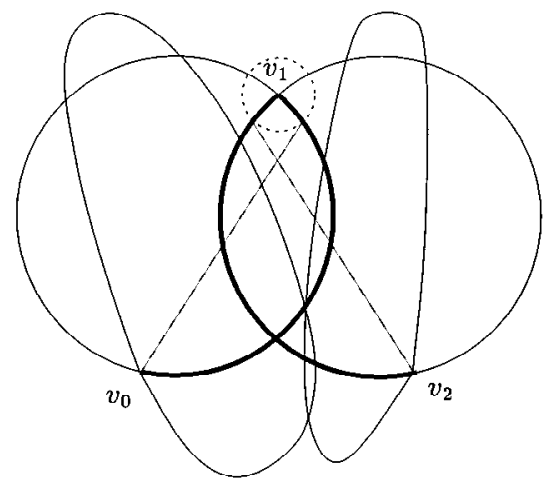

Fig. 4. Illustrating the proof of Lemma 4 for a path of $\Gamma^{R}$.

If $\zeta$ is a path, its total turning angle is, in the above notations,

$$
\sum_{i=1}^{k} a\left(v_{i}\right)+\sum_{i=1}^{k+1} \theta\left(\gamma_{i}\right) \geq k \pi,
$$

which implies that

$$
\sum_{i=1}^{k}\left(\pi-a\left(v_{i}\right)\right) \leq \sum_{i=1}^{k+1} \theta\left(\gamma_{i}\right) .
$$

We now add these inequalities, over all cycles and paths composing $\Gamma^{R}$, and obtain

$$
\sum_{v \in R}(\pi-a(v)) \leq \sum_{\gamma \in \Gamma^{R}} \theta(\gamma) \leq \sum_{\gamma \in \Gamma_{\text {int }}} \theta(\gamma)
$$

as asserted.

\section{Remarks}

(A) In [1] we proved that $|R| \leq 6 n-12$, under the assumption that all vertices of $\mathcal{A}(\mathcal{C})$ are regular. Theorem 1 shows that the same bound holds with the weaker assumption that there are no irregular vertices on the boundary of $U=\cup \mathcal{C}$. (Recall, however, that the result in [1] does not require, as we do, that all members of $\mathcal{C}$ be convex.)

(B) Suppose that any two members of $\mathcal{C}$ have at most $s$ (a constant number) of boundary points in common. How large can $|R|$ be? One can show that, even for $s=4$, the maximum possible value of $|R|$ can be $\Omega\left(n^{4 / 3}\right)$. To see this, take a set $P$ of $n$ points and a set $L$ of $n$ lines, so that there are $\Theta\left(n^{4 / 3}\right)$ incidences between $P$ and $L$ (see Chapter 11 of [3]). Replace each point in $P$ by a disk of radius $\varepsilon$, for some sufficiently small $\varepsilon>0$, and replace each line $\ell \in L$ by a long rectangle whose width is $\varepsilon$ and whose long bottom edge is parallel to $\ell$, lying above $\ell$, and at distance $\varepsilon^{\prime}<\varepsilon$ from it. One can show that, for an appropriate choice of $\varepsilon$ and $\varepsilon^{\prime}$, the number of intersections between any disk and 
any rectangle is at most two, that each incidence between a point of $P$ and a line of $L$ corresponds to an intersecting pair of a disk and a rectangle, and that each intersection point between such a pair lies on the boundary of the union. Hence, we have a collection of $2 n$ disks and rectangles satisfying $|R|=\Theta\left(n^{4 / 3}\right)$. Is this construction asymptotically best possible?

(C) It is not hard to see that the coefficient 2 of the term $|I|$ in Theorem 1 cannot be replaced by any smaller constant. To see this, take $n$ copies of a regular $n$-gon, slightly rotated around their common center, and, for each original vertex, clip the batch of its copies with a small rectangle. This creates $2 n^{2}$ regular vertices on the boundary of the union of the resulting collection of $2 n$ convex sets. On the other hand, $|I|$ is about $n^{2}$. We also note that if $I \neq \emptyset$ then the bound in Theorem 1 is not tight, because we have ignored in (1) all terms $a(v)$ for $v \in I$, so we cannot have equality any more.

\section{Proof of Theorem 2}

Assume without loss of generality that every curve $c$ has a point $p_{c}$ that belongs to the boundary of $U$, the union of the interiors of all family members. Let $q$ be one of the (at most $k$ ) intersection points of two curves, $c$ and $c^{\prime}$. Connect $p_{c}$ to $p_{c^{\prime}}$ by an arc ("edge"), going first from $p_{c}$ to $q$ in clockwise direction around $c$, and then following the boundary of $c^{\prime}$ in counterclockwise direction to $p_{c^{\prime}}$. For each pair $c, c^{\prime}$ of family members that contribute an intersection point $q$ to the boundary of $U$, construct such an edge that connects $p_{c}$ to $p_{c^{\prime}}$ via $q$, but do this for only one such point $q$. The two pieces an edge consists of are called half-edges. It is easy to show that any two half-edges not incident to the same point $p_{c}$ intersect an even number of times. Thus, these edges form a graph drawing with the property that any two edges not incident to the same vertex $p_{c}$ intersect an even number of times. This implies that the underlying graph is planar (see [5] or Corollary 3.1 of [2]), and, since it has no multiple edges, the number of its edges is at most $3 n-6$. The total number of vertices along the boundary of $U$ is obviously at most $k$ times larger than that. To see that the bound is tight, use the same construction as in [1], but replace each pair of intersection points of a pair of boundaries by $k$ consecutive intersections, all lying on the boundary of the union; refer to [1] for more details.

\section{References}

1. K. Kedem, R. Livne, J. Pach, and M. Sharir, On the union of Jordan regions and collision-free translational motion amidst polygonal obstacles, Discrete Comput. Geom. 1 (1986), 59-71.

2. L. Lovász, J. Pach, and M. Szegedy, On Conway's thrackle conjecture, Discrete Comput. Geom. 18 (1997), 369-376.

3. J. Pach and P.K. Agarwal, Combinatorial Geometry, Wiley-Interscience, New York, 1995.

4. S. Whitesides and R. Zhao. $K$-Admissible Collections of Jordan Curves and Offsets of Circular Arc Figures. Technical Report SOCS 90.08, McGill University, Montreal, 1990.

5. Ch. Chojnacki (A. Hanani), Über wesentlich unplättbare Kurven in drei-dimensionalen Raume, Fund. Math. 23 (1934), 135-142.

Received May 5, 1997, and in revised form July 15, 1997. 